\title{
STRUCTURAL COMPLEXITY AND FLUORESCENCE HETEROGENEOUS DECAYS IN PROTEINS
}

\author{
A. Di Venere, G. Mei", N. Rosato, A. Finazzi Agro' \\ Dipartimento di Medicina Sperimentale e Scienze Biochimiche \\ Università di Roma "Tor Vergata" \\ Via di Tor Vergata, 135, Rome 00133, Italy \\ AND G. GILARDI
}

Department of Biochemistry, Imperial College of Science, Technology and Medicine London, UK

\begin{abstract}
Optical spectroscopy is a suitable technique to study the functional and structural properties of large biomolecules in solution without damaging the samples. In particular, measurements of time-resolved fluorescence may give important information on the tertiary structure of proteins. Furthermore, the study of fluorescence depolarization as a function of time is a very common method to follow the dynamics of the local domains and subunits which form the quaternary structure of oligomeric enzyme. The analysis of fluorescence decays in terms of continuous distribution of lifetimes seems to be an appropriate approach to describe the dynamics of proteins which ranges over an enormous time scale (from $10 \mathrm{ps}$ to $10 \mathrm{~ns}$ ). The results obtained for several proteins are reported and discussed. The data provide further confirmation of the correlation which exist between heterogeneous fluorescence decays and a hierarchy of many conformational substates in proteins.
\end{abstract}

PACS numbers: 87.15.Mi

\section{Introduction}

Proteins play a fundamental role in the living organisms. As enzymes they catalyze several chemical reactions of metabolism, supply immunological control and take part in cell's growth, differentiation and death. It is well known that the three-dimensional structure of proteins is strictly correlated to their biological function. Ten years ago, a hierarchical scheme of protein dynamics was discovered [1] and compared to the organized nature of protein structure. It turned out that an enzyme is not a "static" molecule but a rather complex system which

*To whom all correspondence should be addressed. 
fluctuates among several interconverting conformational substates. In particular, the weak interactions among protein subunits and domains provide the structural flexibility which is necessary for their enzymatic activity. These characteristics of proteins tertiary structure may be studied by their intrinsic fluorescence due to tryptophan (trp) and tyrosine (tyr) residues. In particular, time-resolved spectroscopy and anisotropy decay measurements are extremely sensitive methods to reveal the heterogeneity of these systems and to explore the rate at which local structural changes take place.

The fluorescence decay of tryptophan and tyrosine in water can be easily fitted by two exponentials. The indole dynamics is even less complex showing only one discrete component centered around 3 ns. On the other hand, even single tryptophan-containing proteins show complex fluorescence decays which may be fitted only by multiexponential functions. The origin of this complexity may be therefore ascribed to the heterogeneity of the fluorophores' microenvironment due to the presence of the protein matrix. According to this hypothesis, in the last ten years it has been shown that the emission decay of proteins may be better fitted using continuous distributions of fluorescence lifetime instead of the sum of discrete components. According to this approach the full width at half maximum (FWHM) of the distributed lifetimes function is representative of the several microscopic substates which characterize the fluctuating tertiary structure of protein molecules. In order to test this model time-resolved experiments have been performed varying the chemico-physical conditions of the different samples. Interestingly it has been found that the distribution profiles are extremely sensitive to temperature [2], denaturants [3], single aminoacid mutations [4] and ligands binding [5].

In this study we report the results obtained for several proteins using synchrotron radiation or a laser beam as excitation sources. Both single and multiple tryptophan-containing proteins have been analyzed in terms of continuous, Lorentzian-shaped, distribution of lifetimes. The model has been also extended to proteins which contain only tyrosine emitting fluorophores. A comparison between steady state and dynamic fluorescence data has been performed and a clear correlation between the parameters measured has been found. The complexity of the fluorescence decay has been found to depend both on the number of fluorophores present in each sample and on the polarity of their microenvironment. This last result has been also confirmed by protein unfolding experiments. In particular, once that the secondary and tertiary structures are totally destroyed, an evident similarity of fluorescence lifetime distributions among different samples has been observed.

\section{Materials and methods}

Purified protein samples were diluted in standard buffers, namely potassium phosphate, Tris $\mathrm{HCl}$ or Hepes, in the $\mathrm{pH}$ range between 6.0 and 8.0 units.

Absorption in a $1 \mathrm{~cm}$ optical path cuvette was always below 0.2 upon excitation at $280 \mathrm{~nm}$. Samples were always thermostated at $20^{\circ} \mathrm{C}$ during measurements using an external circulating water bath.

To avoid Rayleigh and Raman scattering the emission light was observed through a cut-off filter selected in the range $305-330 \mathrm{~nm}$. In the case of bovine 
superoxide dismutase (BSOD) the glass filter was substituted by a solution of triton X100 in water.

The intrinsic fluorescence decays were measured using frequency domain methods. In particular, phase shift and demodulation techniques were employed in the range 5 to $250 \mathrm{MHz}$ as previously described [6]. The experiments were carried out using the synchrotron radiation source at the ADONE storage ring (PULS laboratory, Frascati; Italy) or a frequency doubled rhodamine $6 \mathrm{G}$ dye laser, Nd-YAG laser-driven (LASP laboratory, Rome, Italy). Excitation wavelengths were selected in the range $280-295 \mathrm{~nm}$ depending on the particular steady state excitation spectrum of each protein.

Fully denaturated samples were obtained after at least $12 \mathrm{~h}$ incubation at $4^{\circ} \mathrm{C}$ in presence of different concentration of urea or guanidinium hydrochloride. The unfolding was checked by circular dichroism titration curves in the peptidic region $200-250 \mathrm{~nm}$.

\section{Results and discussion}

\subsection{Proteins with a single fuorophore}

The results of time-resolved experiments performed on different protein samples are reported in Table I. Interestingly all the single fluorophore-containing proteins show a fluorescence decay which may be easily fitted using a single Lorentzian-shaped function. The only exception reported for comparison is observed for apo-azurin. However, it should be pointed out that the tryptophan residue contained in this molecule has a peculiar structured spectrum which makes apo-azurin a rather unique example in fluorescence literature. In particular, there are several spectroscopic evidences (optical activity and X-ray crystallography) that the fluorophore is totally immobilized and buried in the protein hydrophobic core $[7,8]$. As a matter of fact, when a single aminoacid in the neighbourhood of the fluorophore is substituted by site directed mutagenesis (apo azurin I7S mutant, apo AZ-I7S) the single fluorescence lifetime is transformed in a very heterogeneous decay (Table I). This result was interpreted as a drastic modification of the fluorophore environment due to a possible presence of water molecules [4]. Actually, time-resolved depolarization measurements have revealed an increase in the tryptophan mobility in this sample (data not shown), confirming that an enhanced structural heterogeneity of the protein must be expected in the nanosecond time range. On the other hand, a narrower distribution was measured whenever the influence of the protein matrix was less relevant. This is the case of human superoxide dismutase (HSOD) and BSOD which respectively contain only one tryptophan or tyrosine residue lying on the protein surface. For these samples the fluorescent probe is largely surrounded by the solvent molecules and thus experiences a less heterogeneous variety of structured microstates. It is important to observe that the detected conformational substates are in these cases independent on the particular protein's fluorophore. In fact, both for BSOD, which contains a tyrosine, and for HSOD, which contains a tryptophan, the same value of the FWHM was observed $(\approx 450 \mathrm{ps})$. 
TABLE I

Time-resolved fluorescence parameters of native samples.

\begin{tabular}{l|c|c|c|c|c|c|c|c|c}
\hline \hline Sample & $\begin{array}{c}\text { Number of } \\
\text { fluorophores }\end{array}$ & $\begin{array}{c}\mathrm{CM} \\
{[\mathrm{nm}]}\end{array}$ & $\begin{array}{c}C_{1} \\
{[\mathrm{~ns}]}\end{array}$ & $\begin{array}{c}W_{1} \\
{[\mathrm{~ns}]}\end{array}$ & $\begin{array}{c}F_{1} \\
{[\%]}\end{array}$ & $\begin{array}{c}C_{2} \\
{[\mathrm{~ns}]}\end{array}$ & $\begin{array}{c}W_{2} \\
{[\mathrm{~ns}]}\end{array}$ & $\begin{array}{c}F_{2} \\
{[\%]}\end{array}$ & $\chi^{2}$ \\
\hline Holo HSOD & 1 (per subunit) & 360 & - & - & - & 2.22 & 0.42 & 1.00 & 1.5 \\
Apo HSOD & 1 (per subunit) & 360 & - & - & - & 2.65 & 0.73 & 1.00 & 1.5 \\
Holo BSOD & 1 (per subunit) & 310 & - & - & - & 1.43 & 0.47 & 1.00 & 1.2 \\
Apo BSOD & 1 (per subunit) & 310 & - & - & - & 1.93 & 1.21 & 1.00 & 1.3 \\
Apo AZ & 1 & 325 & - & - & - & 4.70 & - & 1.00 & 2.1 \\
Apo AZ-I7S & 1 & 362 & - & - & - & 3.51 & 2.42 & 1.00 & 1.1 \\
\hline GST & 2 & 342 & 0.78 & 0.06 & 0.78 & 3.11 & 0.05 & 0.22 & 1.3 \\
aEF-1 $\alpha$ & 2 & 350 & 0.22 & 0.21 & 0.12 & 3.42 & 3.30 & 0.88 & 1.2 \\
Apo-AVD & 4 (per subunit) & 352 & 0.99 & 0.93 & 0.47 & 2.60 & 0.23 & 0.53 & 1.6 \\
Holo-AVD & 4 (per subunit) & 341 & 0.98 & - & 0.92 & 2.93 & - & 0.08 & 1.8 \\
CCP & 7 & 334 & 0.25 & 0.39 & 0.73 & 1.62 & 0.93 & 0.27 & 0.8 \\
AAO & 14 (per subunit) & 342 & 0.44 & 0.96 & 0.48 & 2.51 & 0.70 & 0.52 & 0.9 \\
\hline
\end{tabular}

$\mathrm{CM}$ - center of mass of the steady state fluorescence spectrum;

$C_{1}, C_{2}$ - centers of distributions, $\Delta C_{1,2} \approx 0.03 \mathrm{~ns}$;

$W_{1}, W_{2}$ - widths of distributions, $\Delta W_{1,2} \approx 0.04 \mathrm{~ns}$;

$F_{1}, F_{2}$ - relative fluorescence fractions of distributions, $\Delta F_{1,2} \approx 0.01$;

$\chi^{2}$ - reduced chi-squared of phase and modulation data fit;

apo $\mathrm{AZ}$ - apo azurin from pseudonomas aeruginosa;

GST - glutathione transferase;

aEF- $1 \alpha$ - archaebacterium elongation factor;

holo-AVD - holo a vidin.

\subsection{Proteins containing more than one fluorophore}

In the second part of Table I the results relative to multi-tryptophan proteins are reported. The increased complexity of the fluorescence decay is evident as bimodal distributions are required to fit the data. Recently time-resolved experiments have been performed as a function of the emission wavelength in the case of the most complex system, namely ascorbate oxidase (AAO). It has been found (manuscript in preparation) that the several tryptophans contained in this protein may be classified in at least two spectroscopical classes which are indeed compatible with the two components reported in Table I. Quenching experiments have confirmed that the short $(0.44 \mathrm{~ns})$ and the long $(2.51 \mathrm{~ns})$ components may be associated to buried and exposed tryptophans respectively.

In order to test if this result may be generalized to other proteins a general, preliminary correlation between the steady state emission spectrum and the dynamic parameters has been searched. In particular, in Fig. 1 the average lifetime, calculated from the data reported in Table I, has been plotted as a function of the fluorescence spectrum barycenter. Indeed, a non-linear trend, which is independent from protein size, has been found. However, more experimental work must 


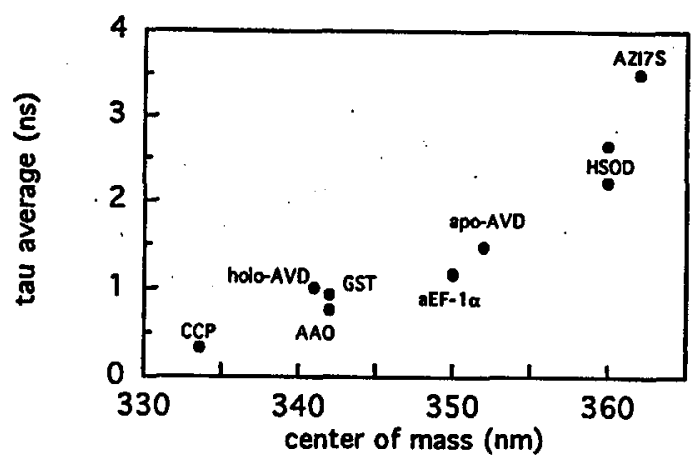

Fig. 1. Dependence of the mean fluorescence lifetime on the steady state emission spectrum center of mass.

be carried out in order to get an accurate chemico-physical explanation for this behaviour. In particular, the dependence of the time-resolved decay on the emission wavelength must be measured for all the other proteins reported in Table I. A generalized model should also take into account solvent relaxation processes as previously suggested [9] and therefore red-edge excitation experiments in the time domain must be performed.

\subsection{Stabilization effect of co-factors and substrates}

An interesting result which arises from the data reported in Table I is the influence that co-factors and substrates have on the heterogeneity of the emission decay. Let us consider the native (holo) and metal-depleted (apo) forms of HSOD and BSOD. In both cases the value of $W_{1}$ is increased upon copper removal without any relevant change in the spectrum profile. This result probably indicates the presence of a larger allowed number of conformational substates due to the loosening of structural rigidity. Again two aspects of dynamic fluorescence methods must be enhanced: first, the result is independent on the particular fluorophore involved; second, the time scale of the spontaneous emission (i.e. of fluorescence lifetimes) perfectly matches that of protein dynamics.

An analogous effect was observed in the case of apo avidin (apo-AVD) which has two tryptophan residues in the active site. In fact, when the protein bounds its biological substrate (vitamin $\mathrm{H}$ ) the bimodal distribution function of the apo-form is reduced to a double exponential decay (Table I). This finding was indeed correlated to a reduced freedom of the indolyl side-chains facing the binding site [5].

\subsection{Similarity of the unfolded states}

The study of the unfolded protein states are a relevant problem in biophysics. They provide a clue for a better understanding of the different mechanisms and interactions which stabilize the native structure. Recently it has been shown that denatured proteins may have significant amounts of local, residual structures [10]. As a consequence, also in these disordered states, the protein matrix might play a significant role on the environment of fluorescent probes. In Table II the data 
TABLE II

Time-resolved fluorescence parameters of unfolded proteins.

\begin{tabular}{l|c|c|c|c|c|c|c}
\hline \hline Sample & $\begin{array}{c}C_{1} \\
{[\mathrm{~ns}]}\end{array}$ & $\begin{array}{c}W_{1} \\
{[\mathrm{~ns}]}\end{array}$ & $\begin{array}{c}F_{1} \\
{[\%]}\end{array}$ & $\begin{array}{c}C_{2} \\
{[\mathrm{~ns}]}\end{array}$ & $\begin{array}{c}W_{2} \\
{[\mathrm{~ns}]}\end{array}$ & $\begin{array}{c}F_{2} \\
{[\%]}\end{array}$ & $\chi^{2}$ \\
\hline Holo HSOD & - & - & - & 3.35 & 0.68 & 1.00 & 1.5 \\
Apo HSOD & - & - & - & 3.41 & 0.69 & 1.00 & 1.5 \\
\hline Apo AVD & - & - & - & 2.81 & 0.97 & 1.00 & 1.4 \\
CCP & 0.64 & 0.34 & 0.20 & 3.06 & 1.42 & 0.80 & 1.8 \\
AAO & 0.01 & 0.67 & 0.11 & 2.87 & 0.89 & 0.89 & 1.1 \\
\hline$C_{1}, C_{2}-$ centers of distributions, $\Delta C_{1,2} \approx 0.03 \mathrm{~ns} ;$ \\
$W_{1}, W_{2}$ - widths of distributions, $\Delta W_{1,2} \approx 0.04 \mathrm{ns;}$ \\
$F_{1}, F_{2}-$ relative fluorescence fractions of distribu- \\
tions, $\Delta F_{1,2} \approx 0.01 ;$ \\
$\chi^{2}-$ reduced chi-squared of phase and modulation \\
data fit.
\end{tabular}

relative to a few protein samples are reported. The general trend observed upon HSOD (holo and apo) and AVD denaturation is the appearance of a distributed component centered around $3 \mathrm{~ns}$. The average width value of these samples ranges from $0.7 \mathrm{~ns}$ to $1.0 \mathrm{~ns}$ confirming a greater heterogeneity than the single exponential decay measured for the indole.

In the case of cytochrome $\mathrm{C}$ peroxidase (CCP) and $\mathrm{AAO}$, which are very complex systems in terms of fluorophores number, a short component is also present. However, the fluorescence fraction is smaller than that of the corresponding native samples indicating the presence of residual structured microdomains which may be due to disulfide bridges.

In conclusion, we believe that time-resolved fluorescence techniques are suitable spectroscopic methods to reveal the conformational substates which characterize protein dynamics. In particular, the distributional analysis is extremely sensitive to the chemico-physical parameters which influence protein structure. A more detailed study as a function of excitation and emission wavelengths is therefore required to provide new information which may be used to monitor protein folding and activity.

\section{Acknowledgments}

We wish to thank the staff of the LASP and the PULS laboratories in which the dynamic experiments were performed.

\section{References}

[1] A. Ansari, S. Berendzen, S.F. Bowne, M. Frauenfelder, I.E. Iben, T.B. Sanke, E. Shymsunder, R. Young, Proc. Natl. Acad. Sci. USA 82, 5000 (1985).

[2] N. Rosato, E. Gratton, G. Mei, A. Finazzi Agro', Biophys. J. 58, 817 (1990).

[3] G. Mei, N. Rosato, N. Silva, R. Rusch, E. Gratton, I. Savini, A. Finazzi Agro', Biochemistry 31, 7224 (1992). 
[4] G. Gilardi, G. Mei, N. Rosato, G.W. Canters, A. Finazzi Agro', Biochemistry 33, 1425 (1994).

[5] G. Mei, L. Pugliese, N. Rosato, L. Toma, M. Bolognesi, A. Finazzi Agro', J. Mol. Biol. 242, 559 (1994).

[6] E. Gratton, M. Limkeman, Biophys. J. 44, 315 (1983 a); E. Gratton, D.M. Jameson, N. Rosato, G. Weber, Rev. Sci. Instrum. 55, 488 (1983 b).

[7] A. Finazzi Agro', G. Rotilio, L. Avigliano, P. Guerrieri, V. Boffi, B. Mondovi', Biochemistry 9, 2009 (1970).

[8] H. Nar, A. Messerschmidt, R. Huber, M. van de Kamp, G.W. Canters, J. Mol. Biol. 221, 765 (1991).

[9] A.P. Demchenko, in: Ultraviolet Spectroscopy of Proteins, Springer-Verlag, Berlin 1986, p. 158.

[10] K.A. Dill, D. Shortle, Ann. Rev. Biochem. 60, 795 (1991). 